\title{
Generation of endogenous hydrogen sulfide by cystathionine $\gamma$-lyase limits renal ischemia/reperfusion injury and dysfunction
}

\author{
Pinpat Tripatara ${ }^{1,2,6}$, Nimesh SA Patel ${ }^{1,6}$, Massimo Collino ${ }^{3}$, Margherita Gallicchio ${ }^{3}$, Julius Kieswich ${ }^{1}$, Sara Castiglia ${ }^{3}$, \\ Elisa Benetti ${ }^{3}$, Keith N Stewart ${ }^{4}$, Paul AJ Brown ${ }^{5}$, Mohammed M Yaqoob ${ }^{1}$, Roberto Fantozzi ${ }^{3}$ and \\ Christoph Thiemermann ${ }^{1}$
}

The generation of endogenous hydrogen sulfide may either limit or contribute to the degree of tissue injury caused by ischemia/reperfusion. A total of 74 male Wistar rats were used to investigate the effects of endogenous and exogenous hydrogen sulfide in renal ischemia/reperfusion. Administration of the irreversible cystathionine $\gamma$-lyase (CSE) inhibitor, $\mathrm{dL}$-propargylglycine, prevented the recovery of renal function after $45 \mathrm{~min}$ ischemia and $72 \mathrm{~h}$ reperfusion. The hydrogen sulfide donor sodium hydrosulfide attenuated the (renal, tubular, and glomerular) dysfunction and injury caused by $45 \mathrm{~min}$ ischemia and $6 \mathrm{~h}$ reperfusion. Western blot analysis of kidneys taken at $30 \mathrm{~min}$ reperfusion showed that sodium hydrosulfide significantly attenuated phosphorylation of mitogen-activated protein kinases ( $\mathrm{p}$-38, c-JUN N-terminal protein kinase $1 / 2$, and extracellular signal-regulated kinase 1/2) and activation of nuclear factor- $\kappa \mathrm{B}$. At $6 \mathrm{~h}$ reperfusion, sodium hydrosulfide significantly attenuated the histological score for acute tubular necrosis, the activation of caspase-3 and Bid, the decline in the expression of anti-apoptotic $\mathrm{BCl}-2$, and the expression of nuclear factor- $\mathrm{BB}$-dependent proteins (inducible nitric oxide synthase, cyclo-oxygenase-2, and intercellular adhesion molecule-1). These findings suggest that (1) the synthesis of endogenous hydrogen sulfide by CSE is essential to protect the kidney against ischemia/reperfusion injury and dysfunction and aids in the recovery of renal function following ischemia/reperfusion, (2) hydrogen sulfide generated by sodium hydrosulfide reduces ischemia/reperfusion injury and dysfunction, and morphological changes of the kidney, and (3) the observed protective effects of hydrogen sulfide are due to both anti-apoptotic and anti-inflammatory effects.

Laboratory Investigation (2008) 88, 1038-1048; doi:10.1038/labinvest.2008.73; published online 4 August 2008

KEYWORDS: kidney; ischemia/reperfusion; cystathionine $\gamma$-lyase; hydrogen sulfide; dL-propargylglycine

Hydrogen sulfide $\left(\mathrm{H}_{2} \mathrm{~S}\right)$ is a membrane-permeable, gaseous mediator, which has specific cellular and molecular targets. ${ }^{1}$ $\mathrm{H}_{2} \mathrm{~S}$ is synthesized through desulphhydration of cysteine that is mainly catalyzed by the pyridoxal- $5^{\prime}$-phosphate-dependent enzymes, cystathionine $\beta$-synthase (CBS) and cystathionine $\gamma$-lyase (CSE). Owing to differences in tissue distribution, CBS is the predominant source of $\mathrm{H}_{2} \mathrm{~S}$ in the nervous system, whereas CSE is the predominant source of $\mathrm{H}_{2} \mathrm{~S}$ in the cardiovascular system. ${ }^{1}$
Although initially perceived as being merely a toxic gas ${ }^{2}$ that causes $85 \%$ mortality at high concentrations, ${ }^{3} \mathrm{H}_{2} \mathrm{~S}$ has been reported to have complex roles (both detrimental and beneficial) in biology, especially in the central nervous system and cardiovascular system. $\mathrm{H}_{2} \mathrm{~S}$ is known as a neuromodulator as well as an intracellular neurological messenger. ${ }^{4}$ It activates NMDA receptors, regulates the redox status, maintains the excitatory/inhibitory balance in neurotransmission, and inhibits oxidative damage through scavenging free

\footnotetext{
${ }^{1}$ Centre for Translational Medicine and Therapeutics, William Harvey Research Institute, St Bartholomew's and The Royal London School of Medicine and Dentistry, Queen Mary University of London, London, UK; ${ }^{2}$ Department of Pharmacology, Faculty of Medicine, Siriraj Hospital, Bangkok, Thailand; ${ }^{3}$ Department of Anatomy, Pharmacology and Forensic Medicine, University of Turin, Turin, Italy; ${ }^{4}$ Department of Medicine and Therapeutics, University of Aberdeen, Aberdeen, UK and ${ }^{5}$ Department of Pathology, University of Aberdeen, Aberdeen, UK

Correspondence: Dr NSA Patel, PhD, Centre for Translational Medicine and Therapeutics, William Harvey Research Institute, St Bartholomew's and The Royal London School of Medicine and Dentistry, Queen Mary University of London, Charterhouse Square, London EC1M 6BQ, UK.

E-mail: n.s.patel@qmul.ac.uk

${ }^{6}$ These authors contributed equally to this work.

Received 14 March 2008; revised 06 June 2008; accepted 14 June 2008
} 
radicals and reactive species. In fact, inhibitors of $\mathrm{H}_{2} \mathrm{~S}$ synthesis have shown to reduce the infarct volume caused by middle cerebral artery occlusion. ${ }^{5}$ In contrast however, endogenous $\mathrm{H}_{2} \mathrm{~S}$ is produced by myocardial ischemia in sufficient amounts to limit myocardial tissue injury ${ }^{6,7}$ and impairment of $\mathrm{H}_{2} \mathrm{~S}$-negative-regulated $\beta$-adrenergic function during ischemia may induce cardiac arrhythmias. ${ }^{8}$ In addition, generation of $\mathrm{H}_{2} \mathrm{~S}$ by $\mathrm{H}_{2} \mathrm{~S}$ donors is beneficial in animal models of hypertension, ${ }^{9-11}$ whereas inhibition of the endogenous synthesis of $\mathrm{H}_{2} \mathrm{~S}$ is beneficial in endotoxic and hemorrhagic shock. ${ }^{12-14}$ Moreover, transfecting a recombinant-defective adenovirus containing the CSE gene into human aorta smooth muscle cells stimulated apoptosis, ${ }^{15}$ whereas $\mathrm{H}_{2} \mathrm{~S}$ promoted the survival of neutrophils through inhibition of apoptotic pathways, caspase- 3 cleavage and p38 mitogen-activated protein kinase (MAPK) phosphorylation. ${ }^{16}$ Further contradictory data for the role of $\mathrm{H}_{2} \mathrm{~S}$ in the pathophysiology of inflammation has also been raised. $\mathrm{H}_{2} \mathrm{~S}$ has been reported to be a mediator of lipopolysaccharide-induced inflammation, ${ }^{17}$ however, $\mathrm{H}_{2} \mathrm{~S}$ can also produce an anti-inflammatory effect against lipopolysaccharide, in gastrointestinal, ${ }^{18}$ and neurological systems. ${ }^{19}$ Surprisingly, an effect of $\mathrm{H}_{2} \mathrm{~S}$ on the inhibition of cytochrome $c$ oxidase, which has previously been reported to cause toxicity, ${ }^{20}$ led to the discovery of a suspended animation-like state in which the oxygen consumption and metabolic rate of mice decreased ${ }^{21}$ that could potentially be of benefit for ischemiarelated conditions.

Although various effects of $\mathrm{H}_{2} \mathrm{~S}$ in disease have been discovered, the effects of $\mathrm{H}_{2} \mathrm{~S}$ in ischemia/reperfusion injury (IRI) of the kidney are unknown. Thus, we have investigated the effects of endogenous $\mathrm{H}_{2} \mathrm{~S}$ on the recovery of renal function following IRI and the effects of exogenous $\mathrm{H}_{2} \mathrm{~S}$ on renal function following IRI.

\section{MATERIALS AND METHODS Animal Care}

A total of 74 male Wistar rats (200-320 g) (Charles River Ltd, Margate, UK) received a standard diet and water ad libitum, and were cared for in accordance with both the UK Home Office Guidance in the Operation of the Animals (Scientific Procedures) Act 1986, published by Her Majesty's Stationery Office, London, UK and the Guide for the Care and Use of Laboratory Animals, published by the American Physiological Society.

\section{Renal Ischemia/Reperfusion: Recovery Model}

As homozygous knockout mice for CSE are not viable, ${ }^{22}$ we chose to inhibit the activity of CSE by using the irreversible CSE inhibitor dL-propargylglycine (PAG). Blood samples were collected from the tail veins of rats before the experiment. Rats were anesthetized $(1.5 \mathrm{ml} / \mathrm{kg}$, i.p. $)$ with a ketamine $(100 \mathrm{mg} / \mathrm{ml})$ and xylazine $(20 \mathrm{mg} / \mathrm{ml})$ mixture $(2: 1)$ and anesthesia was maintained for the duration of ischemia (45 min). Rats were randomly divided into four groups and treated with either saline (IRI, $1 \mathrm{ml} / \mathrm{kg}$ i.p., $N=8$ ) or PAG (IRI PAG, $50 \mathrm{mg} / \mathrm{kg}, 1 \mathrm{ml} / \mathrm{kg}$ i.p., $1 \mathrm{~h}$ before ischemia, $N=9$ ). Bilateral renal occlusion was performed by clamping the renal pedicles for $45 \mathrm{~min}$ with nontraumatic arterial clamps, which was followed by reperfusion for $72 \mathrm{~h}$ as previously described. ${ }^{23}$ Sham-operated rats underwent identical surgical procedures to rats undergoing IRI except that arterial clamps were not applied in the absence (Sham, $N=4$ ) or presence of PAG (Sham PAG, $N=4$ ). The dose of PAG used was based on that previously shown to increase infarct size in a rat model of myocardial injury. ${ }^{6}$

\section{Renal Ischemia/Reperfusion: Nonrecovery Model}

Rats were anesthetized with sodium thiopentone (110 mg/kg i.p.) and anesthesia was maintained by supplementary injections ( $\sim 10 \mathrm{mg} / \mathrm{kg}$ i.v.) of sodium thiopentone for the duration of the experiment. Rats were randomly divided and treated with either saline (IRI, $2 \mathrm{ml} / \mathrm{kg}, N=12$ ) or NaHS (IRI NaHS, $100 \mu \mathrm{mol} / \mathrm{kg}, 2 \mathrm{ml} / \mathrm{kg}, N=10$ ) administered topically onto the kidneys $15 \mathrm{~min}$ before ischemia and $5 \mathrm{~min}$ before reperfusion. Bilateral renal occlusion was performed by clamping the renal pedicles for $45 \mathrm{~min}$ with nontraumatic arterial clamps, which was followed by reperfusion for $6 \mathrm{~h}$ as previously described. ${ }^{25}$ Sham-operated rats underwent identical surgical procedures to rats undergoing IRI except that microvascular clamps were not applied in the absence (Sham, $N=10$ ) or presence of topical NaHS treatment (Sham NaHS, $N=8$ ). All groups received a continuous infusion of $0.9 \%(\mathrm{w} / \mathrm{v})$ saline $(2.4 \mathrm{ml} / \mathrm{kg} / \mathrm{h}$, i.v. $)$ throughout the reperfusion period. The dose of NaHS used was based on that previously shown to attenuate inflammatory injury in the rat. ${ }^{24}$

Three additional groups of rats were used to investigate the effect of NaHS on intracellular signaling pathways during $45 \mathrm{~min}$ ischemia and $30 \mathrm{~min}$ reperfusion-IRI $(N=3)$, IRI $\mathrm{NaHS}(N=3)$, and Sham $(N=3)$.

\section{Measurement of Biochemical Parameters}

At the end of the reperfusion period, $1 \mathrm{ml}$ blood samples were collected from anesthetized animals. The samples were centrifuged $(6000 \mathrm{~g}$ for $3 \mathrm{~min})$ to separate plasma/serum. All plasma/serum samples were frozen and stored at $-80^{\circ} \mathrm{C}$ and analyzed for biochemical parameters as previously described. ${ }^{25,26}$

\section{Histological Evaluation}

Histology and histological scoring of renal sections for acute tubular necrosis (ATN) were performed as previously described and used for the assessment of renal IRI. ${ }^{23}$ Briefly, 100 intersections were examined for each kidney and a score from 0 to 3 was given for each tubular profile involving an intersection: 0; normal histology, 1; tubular cell swelling, brush border loss, nuclear condensation, with up to one-third of tubular profile showing nuclear loss, 2; as for score 1, but greater than one-third and less than two-third of tubular 
profile shows nuclear loss and 3; greater than two-third of tubular profile shows nuclear loss. The total score for each kidney was calculated by addition of all 100 scores with a maximum score of 300 .

\section{Immunofluorescence for Activated Caspase-3}

Immunofluorescence was performed as previously described. ${ }^{27}$ Briefly, antigen unmasking by autoclaving of histological sections for $10 \mathrm{~min}$ in $10 \mathrm{mM}$ sodium citrate $(\mathrm{pH}$ 6.0) was performed before immunostaining with antibody against cleaved caspase-3 (Asp175, no. 9661, 1:100; Cell Signalling Technology, Danvers, MA, USA). Immune complexes were detected with Alexa Fluor ${ }^{\circledR} 488$ goat anti-rabbit IgG (1:300; Molecular Probe, Eugene, OR, USA). The observation was performed under a Biozero BZ-8000 fluorescent microscope (Keyence, Osaka, Japan) using the BZ Viewer 2.5 under a magnification of $\times 100$. A total of 10 fields were selected at random and the number of cleaved caspase-3-positive cells was counted.

\section{Western Blot Analysis for Apoptotic and Inflammatory Markers}

Tissue extraction of kidney samples was carried out as previously described above. ${ }^{28}$ Briefly, to determine the nuclear translocation of p65 subunit of NF- $\kappa \mathrm{B}$, and hence the activation of NF- $\kappa \mathrm{B}$, both cytosolic and nuclear extractions were used. Determinations of all other proteins-Bid, Bcl-2, iNOS, COX-2, ICAM-1, phosphorylated p38, JNK1/2, and ERK1/2 -were made using only the cytosolic fraction. Approximately $60 \mu \mathrm{g}$ total protein was loaded. Proteins were separated on $8 \%$ (iNOS, COX-2, ICAM-1), $10 \%$ (NF- $\kappa \mathrm{B}$ p65, phosphorylated $\mathrm{p} 38$, JNK1/2, and ERK1/2) or $15 \%$ (Bid, Bcl-2) sodium dodecyl sulfate-polyacrylamide gel electrophoresis and transferred to a polyvinylidene fluoride membrane, which was then incubated with SuperBlock blocking buffer (Pierce Biotechnology Inc., Rockford, IL, USA). Membranes were incubated with primary antibody (rabbit anti-Bid, Bcl-2, iNOS, COX-2, goat anti-ICAM-1, mouse anti-phosphorylated p38, JNK1/2, ERK1/2 and NF- $\kappa$ B p65). Blots were then incubated with secondary antibody conjugated with horseradish peroxidase and developed with the ECL detection system. The immunoreactive bands were visualized by autoradiography and the density of the bands was evaluated densitometrically using the Gel Pro ${ }^{\circledR}$ Analyzer 4.5, 2000 software (Media Cybernetics, Silver Spring, MD, USA). The membranes were stripped and incubated with $\beta$-actin monoclonal antibody and subsequently with secondary antimouse antibody to assess gel-loading homogeneity.

\section{Materials}

Unless otherwise stated, all compounds used in this study were purchased from Sigma-Aldrich Company Ltd (Poole, Dorset, UK). All solutions used were prepared using nonpyrogenic saline $(0.9 \%(\mathrm{w} / \mathrm{v}) \mathrm{NaCl}$; Baxter Healthcare Ltd, Thetford, Norfolk, UK). Rabbit polyclonal antibodies against
iNOS and apoptosis markers, goat polyclonal antibody against ICAM-1, mouse monoclonal antibody against p65 NF- $\kappa \mathrm{B}$, and horseradish peroxidase-conjugated donkey antigoat and anti-rabbit IgG were from Santa Cruz Biotechnology (Santa Cruz, CA, USA). Rabbit polyclonal antibody against COX-2 was from the Cayman Chemical Co. (Ann Arbor, MI, USA). Antibodies against the phosphorylated form of $\mathrm{p} 38$, JNK1/2, and ERK1/2 MAPKs were obtained from Cell Signaling Technology (Beverly, MA, USA). The anti-mouse IgG horseradish peroxidase-linked whole antibody and Luminol ECL detection reagents were from Amersham (Little Chalfont, Buckinghamshire, UK).

\section{Statistical analysis}

All values described in the text and figures are expressed as mean \pm s.e.m. for $N$ observations. Each data point obtained from up to 12 separate animals. One-way analysis of variance with Dunnett's post hoc test was performed on all data (GraphPad Prism version 4.03 for Windows, GraphPad Software, San Diego, CA, USA, www.graphpad.com). A $P$-value of less than 0.05 was considered to be significant.

\section{RESULTS}

Inhibition of Endogenous $\mathrm{H}_{2} \mathrm{~S}$ Synthesis by CSE Impairs the Recovery of Renal Function after IRI in Rats

After $72 \mathrm{~h}$ of reperfusion, the levels of serum creatinine and urea in rats that underwent $45 \mathrm{~min}$ of renal ischemia had returned to levels that were not significantly different from those in sham-operated rats, indicating a complete recovery of renal function (Figure 1). Compared to rats subjected to IRI only, pretreatment of rats with PAG ( $1 \mathrm{~h}$ before ischemia) significantly increased serum creatinine and urea (Figure 1).

Administration of PAG to sham-operated rats had no effect on any of the biochemical markers measured. There were no significant differences in baseline values of serum creatinine (Sham $34.8 \pm 1.0, N=4$; Sham PAG 35.9 \pm 0.7 , $N=4$; IRI $36.2 \pm 0.9, N=8$; IRI PAG $39.1 \pm 2.3, N=9$ ) or serum urea (Sham $6.15 \pm 0.27, N=4$; Sham PAG $5.40 \pm 0.28$, $N=4$, IRI $6.29 \pm 0.33, N=8$; IRI PAG $6.41 \pm 0.42, N=9$ ).

\section{Exogenous $\mathrm{H}_{2} \mathrm{~S}$ Attenuates the Renal Dysfunction and Injury Caused by IRI in Rats}

When compared to sham-operated rats, rats that underwent $45 \mathrm{~min}$ renal ischemia and $6 \mathrm{~h}$ reperfusion exhibited a significant increase in serum creatinine and aspartate aminotransferase (AST), attenuation in creatinine clearance (CCL) and urine flow, and increase in fractional excretion of $\mathrm{Na}^{+}\left(\mathrm{FE}_{\mathrm{Na}+}\right)$ indicating renal dysfunction, reperfusion injury, glomerular dysfunction, and tubular dysfunction, respectively (Figure 2). Compared to rats subjected to IRI only, pretreatment with NaHS (15 min before ischemia and 5 min before reperfusion) significantly attenuated the effects of IRI on serum creatinine, serum AST, CCL and $\mathrm{FE}_{\mathrm{Na}+}$, and modestly improved urine flow, but this effect was not significant (Figure 2). Administration of NaHS to 

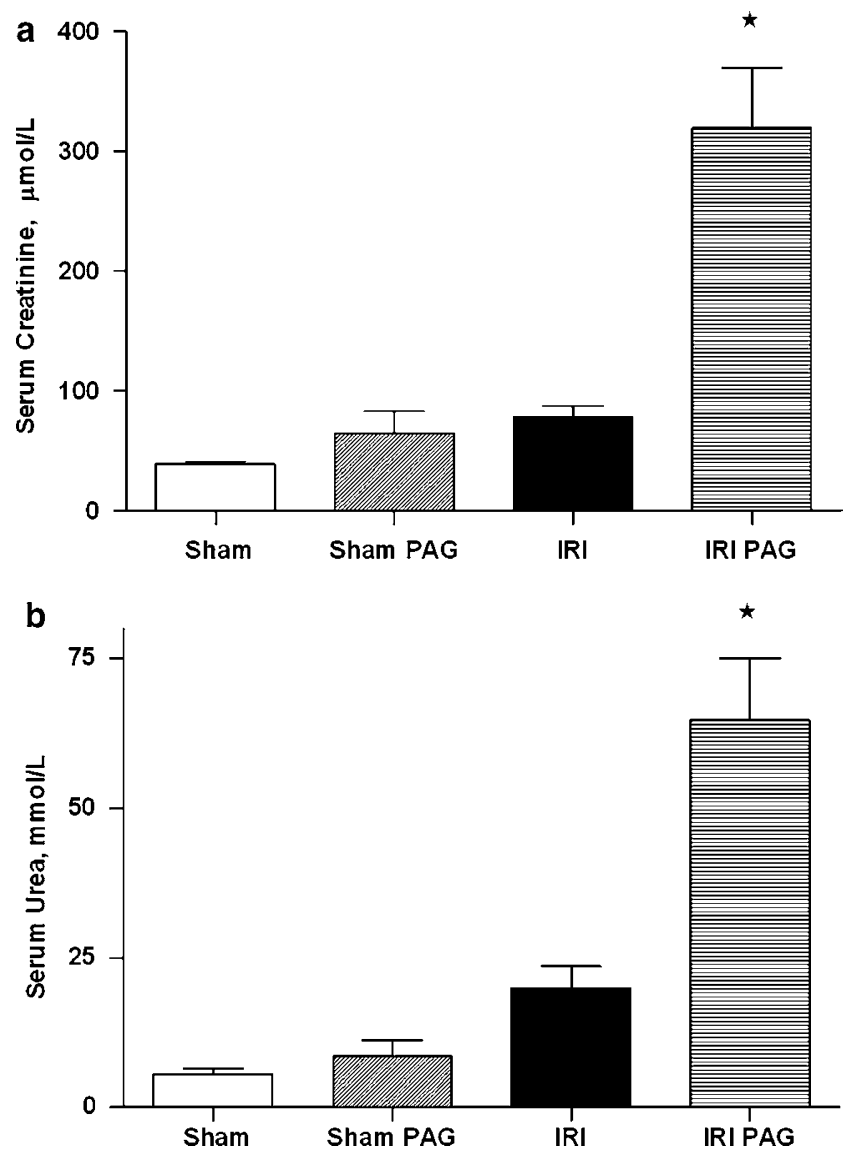

Figure 1 Inhibition of endogenous $\mathrm{H}_{2} \mathrm{~S}$ synthesis with PAG impairs the recovery of renal function after IRI in rats. (a) Serum creatinine and (b) serum urea were measured subsequent to sham operation (Sham, $N=4$ ), renal IRI ( $45 \mathrm{~min}$ ischemia followed by $72 \mathrm{~h}$ reperfusion) (IRI, $N=8$ ), or renal IRI with PAG $(50 \mathrm{mg} / \mathrm{kg}, 1 \mathrm{ml} / \mathrm{kg}$, i.p.) administered $1 \mathrm{~h}$ before ischemia (IRI PAG, $N=9$ ). A separate group of rats subjected to sham IRI received PAG (Sham PAG, $N=4$ ) same dose and time as IRI PAG. Data are expressed as means \pm s.e.m. for $N$ number of observations. ${ }^{*} P<0.05$ vs IRI.

sham-operated rats had no effect on any of the biochemical markers measured $v s$ sham-operated rats.

\section{Exogenous $\mathrm{H}_{2} \mathrm{~S}$ Reduces the Histological Signs of Injury Caused by IRI in Rats}

When compared with normal tubular histology in shamoperated rats (Figure 3a), animals that underwent renal IRI demonstrated the recognized features of severe acute tubular damage (Figure 3d). These features included brush border loss, nuclear condensation, cytoplasmic swelling, and consistent loss of significant numbers of nuclei from tubular profiles (Figure 3d). In contrast, renal sections obtained from rats subjected to IRI and treated with NaHS demonstrated a marked reduction of the histological signs of renal injury (Figure 3c).

On comparison with the histological score for ATN measured from kidneys obtained from sham-operated rats, renal IRI produced a significant increase in histological score
(Figure 3e), indicating necrosis of tubular cells. Administration of NaHS significantly reduced the histological ATN score when compared to that obtained from rats subjected to renal IRI only (Figure 3e), indicating a reduction in renal tubular cell necrosis.

\section{Exogenous $\mathrm{H}_{2} \mathrm{~S}$ Prevents Activation of Caspase-3 and the Decline in the Expression of the Apoptotic Markers Bid and Bcl-2 Caused by IRI in Rats}

When compared to sham-operated rats, renal IRI produced a significant increase in the activation of caspase-3 (Figure 4a), indicating the development of apoptosis. Administration of NaHS significantly reduced the activation of caspase- 3 when compared to that obtained from rats subjected to renal IRI only (Figure 4a), indicating a reduction in apoptosis.

Using an antibody against the intact form of the proapoptotic protein Bid, western blot analysis revealed that kidneys obtained from rats subjected to IRI showed a significant reduction in Bid expression, when compared to sham-operated rats (Figure $4 \mathrm{~b}$ ), thus demonstrating its activation by cleavage of intact Bid into truncated forms of Bid. The administration of NaHS prevented the IRI-induced activation of Bid, when compared to $45 \mathrm{~min}$ ischemia control (Figure 4c). The basal expression of Bcl-2 protein, a wellknown suppressor of apoptosis, was also significantly reduced by IRI (Figure 4c), and this effect was abolished by exogenous NaHS (Figure 4c).

\section{Exogenous $\mathrm{H}_{2} \mathrm{~S}$ Prevents the Increase in Phosphorylation of MAPKs p-38, JNK1/2, and ERK1/2 Caused by IRI in Rats}

When compared to sham-operated rats, kidneys obtained from rats subjected to IRI demonstrated a significant increase in the phosphorylation of p-38 (Figure 5a), JNK1/2 (Figure 5b), and ERK1/2 (Figure 5c). This increase was significantly attenuated in kidneys of rats subjected to IRI that had been treated with NaHS (Figures $5 \mathrm{a}-\mathrm{c}$ ).

\section{Exogenous $\mathrm{H}_{2} \mathrm{~S}$ Prevents the Nuclear Translocation of the NF- $\kappa$ B p65 Subunit and on the Expression of iNOS, COX-2, and ICAM-1 Caused by IRI in Rats}

When compared to sham-operated rats, kidneys obtained from rats subjected to IRI demonstrated a significant increase in the nucleus/cytosol ratio of the NF- $\kappa \mathrm{B}$ p 65 subunit. The rise in this ratio suggested the translocation of p65 to the nucleus (activation of NF- $\kappa \mathrm{B}$ ) (Figure 6a). When compared to IRI control, administration of NaHS attenuated the rise in ratios, hence, indicating reduced activation of NF- $\kappa \mathrm{B}$ (Figure 6a).

When compared to sham-operated rats, kidneys obtained from rats subjected to IRI, demonstrated significant increases in the expression of iNOS (Figure 6b), COX-2 (Figure 6c) and ICAM-1 (Figure 6d). When compared to IRI control, the expression in all three proteins was attenuated in 

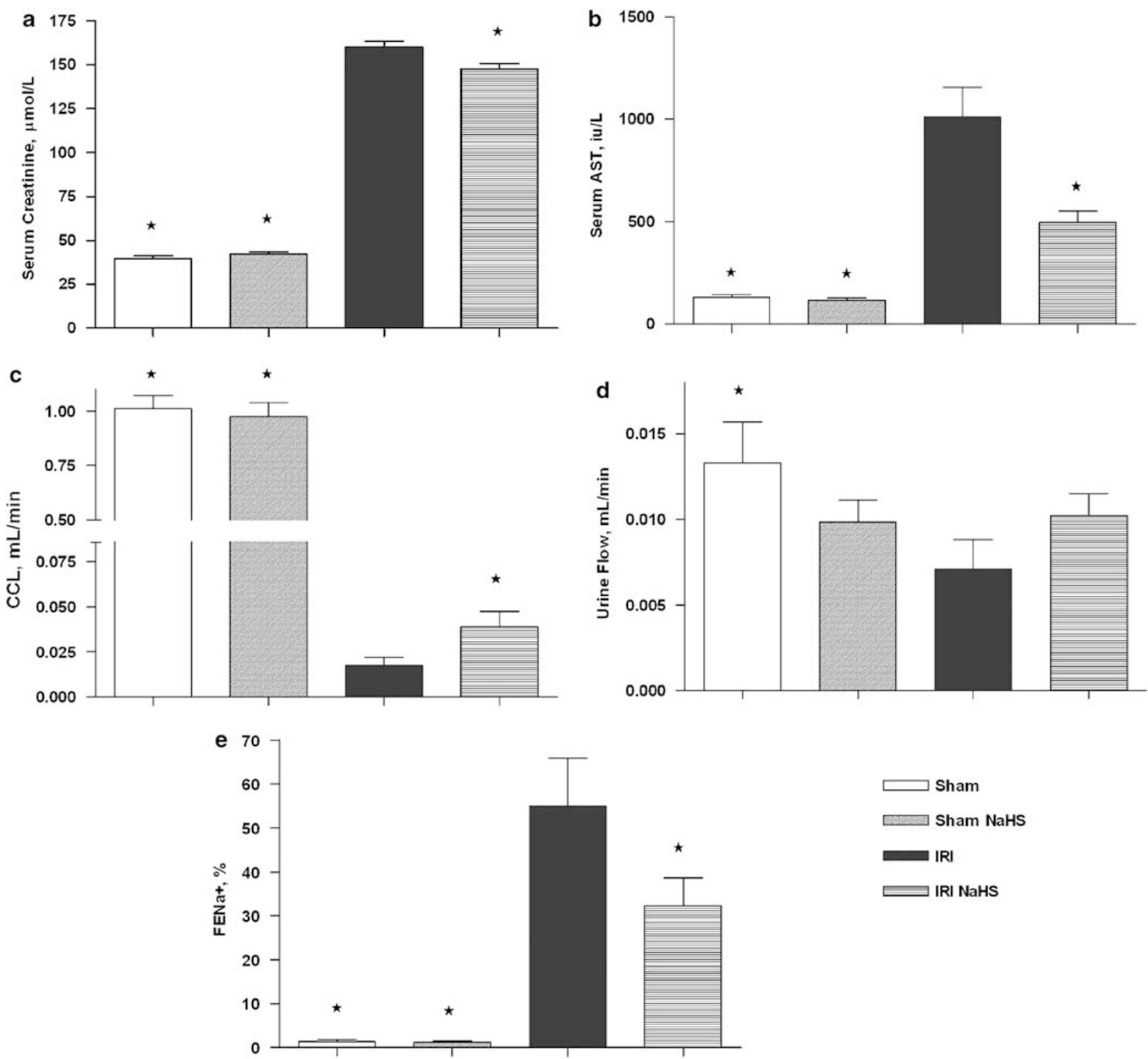

Figure 2 Exogenous $\mathrm{H}_{2} \mathrm{~S}$ attenuates the renal dysfunction and injury caused by IRI in rats. (a) Serum creatinine, (b) serum aspartate aminotransferase (AST), (c) creatinine clearance (CCL), (d) urine flow, and (e) fractional excretion of $\mathrm{Na}^{+}\left(\mathrm{FE}_{\mathrm{Na}_{+}}\right)$were measured subsequent to sham operation (Sham, $\left.N=10\right)$, renal IRI (IRI, $N=12)$, or renal IRI with NaHS $(100 \mu \mathrm{mol} / \mathrm{kg}, 2 \mathrm{ml} / \mathrm{kg}$, onto kidneys) administered $15 \mathrm{~min}$ before $45 \mathrm{~min}$ ischemia and $5 \mathrm{~min}$ before $6 \mathrm{~h}$ reperfusion (IRI NaHS, $N=10$ ). A further group of rats subjected to sham IRI received NaHS, same doses and times as IRI NaHS (Sham NaHS, $N=8$ ). Data are expressed as means \pm s.e.m. for $N$ number of observations. ${ }^{\star} P<0.05$ vs IRI.

kidneys of rats subjected to IRI subsequent to administration of NaHS (Figures 6b-d).

\section{DISCUSSION}

To the best of our knowledge, there is no information relating to the role of $\mathrm{H}_{2} \mathrm{~S}$ in renal IRI. $\mathrm{H}_{2} \mathrm{~S}$ formation is predominantly catalyzed by the pyridoxal-5' -phosphate dependent enzymes, CSE in the cardiovascular system. ${ }^{1}$ We report here for the first time that (1) the synthesis of endogenous $\mathrm{H}_{2} \mathrm{~S}$ by CSE is essential for the recovery of renal function and integrity following IRI and (2) the $\mathrm{H}_{2} \mathrm{~S}$-donor NaHS reduces the renal injury and dysfunction caused by renal IRI in rats.

Specifically, we demonstrate that the CSE inhibitor PAG does not affect renal function when administered to shamoperated animals. Interestingly, after $72 \mathrm{~h}$ of recovery, the irreversible inhibition of CSE not only impaired the recovery of renal function, but also increased the renal dysfunction caused by IRI. This observation suggests that, endogenous $\mathrm{H}_{2} \mathrm{~S}$ synthesis catalyzed by CSE is essential for the recovery from renal dysfunction caused by IRI in rats and favors the 

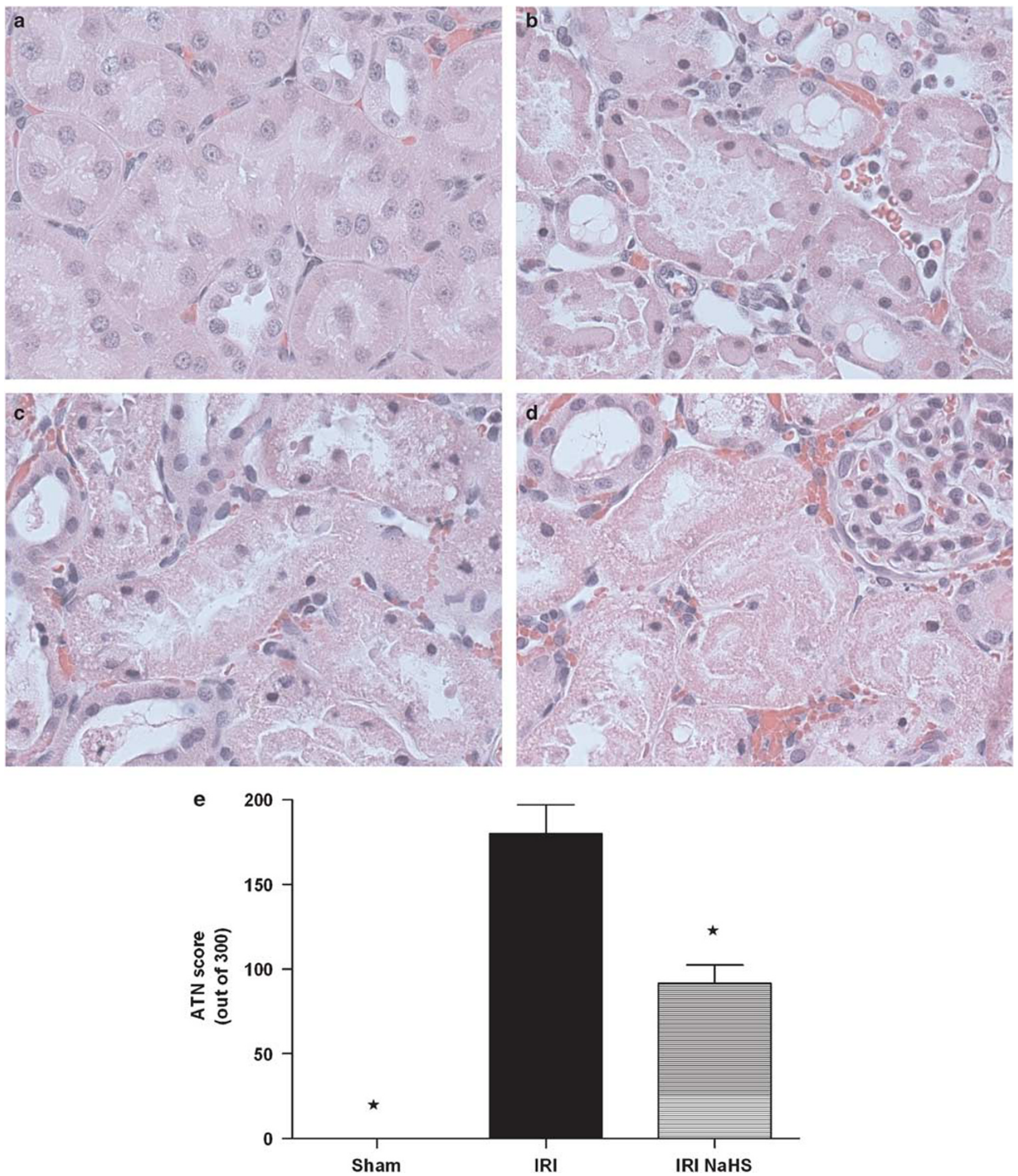

Figure 3 Exogenous $\mathrm{H}_{2} \mathrm{~S}$ reduces the histological signs of injury caused by IRI in rats. Histology of acute tubular necrosis (ATN) (a) score 0: a normal proximal tubule; (b) score 1: tubules revealing brush border loss, nuclear condensation, and cytoplasmic swelling, and loss of nuclei up to one-third of the tubular profile; (c) score 2: more severe cellular loss, with between one-third and two-thirds of the tubular profile denuded of nuclei, and (d) score 3: greater than two-thirds of nuclei loss. Hematoxylin and eosin, figures are representative of at least three experiments performed on different days. (e) ATN score out of a total of 300 (see 'Materials and Methods') subsequent to sham operation (Sham, $N=3$ ), renal IRI (IRI, $N=4)$, or renal IRI with NaHS (100 $\mu \mathrm{mol} / \mathrm{kg}, 2 \mathrm{mI} / \mathrm{kg}$, onto kidneys) administered $15 \mathrm{~min}$ before $45 \mathrm{~min}$ ischemia and $5 \mathrm{~min}$ before $6 \mathrm{~h}$ reperfusion (IRI NaHS, $N=5$ ). Data are expressed as means \pm s.e.m. for $N$ number of observations. ${ }^{*} P<0.05$ vs IRI. 

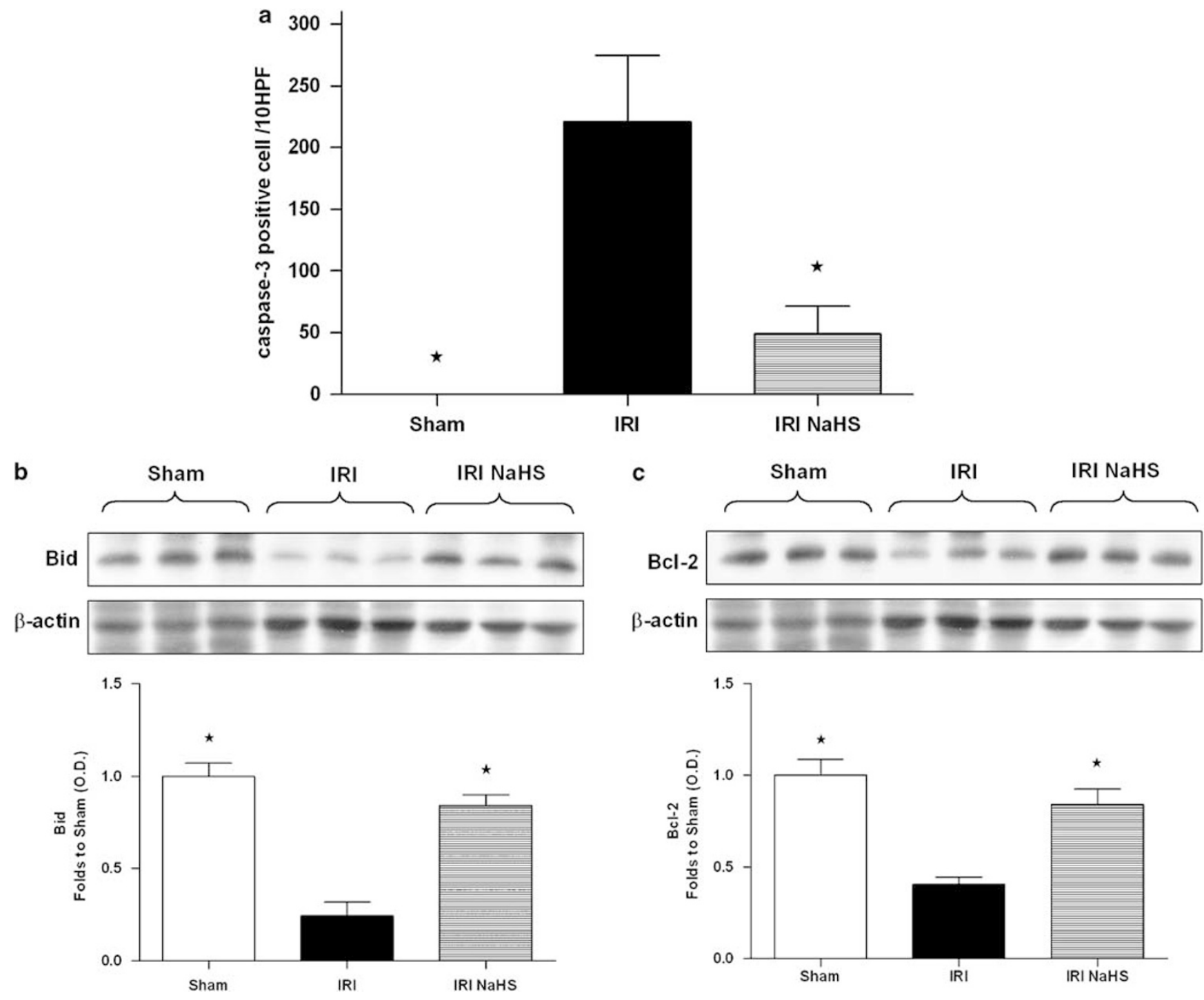

Figure 4 Exogenous $\mathrm{H}_{2} \mathrm{~S}$ prevents caspase-3 activation and the decline in the expression of the apoptotic markers Bid and Bcl-2 caused by IRI in rats. (a) Number of apoptotic cells per 10 high powered fields (HPF) in histological sections processed under immunofluorescence for caspase-3, and western blot analyses of (b) Bid and (c) Bcl-2 expression in kidney homogenates. Histological sections and kidney homogenates were taken from sham-operated rats (Sham), rats subjected to renal IRI (IRI), or rats subjected to renal IRI with NaHS ( $100 \mu \mathrm{mol} / \mathrm{kg}, 2 \mathrm{ml} / \mathrm{kg}$, onto kidneys) administered $15 \mathrm{~min}$ before $45 \mathrm{~min}$ ischemia and $5 \mathrm{~min}$ before $6 \mathrm{~h}$ reperfusion (IRI NaHS). Densitometric analysis of the related bands is expressed as relative optical density (OD), corrected for the corresponding $\beta$-actin contents and normalized using the related sham-operated bands. Densitometric data are expressed as means \pm s.e.m. and each immunoblot is representative of three separate experiments. ${ }^{*} P<0.05$ vs IRI. Note that we used an antibody against the intact form of Bid. Thus, its reduction demonstrates Bid activation by cleavage of intact Bid into truncated forms of tBid.

conclusion that CSE is responsible for the production of $\mathrm{H}_{2} \mathrm{~S}$ in the kidney during IRI.

We used a nonrecovery model of renal IRI in the rat to investigate the effects of $\mathrm{H}_{2} \mathrm{~S}$-donor NaHS on injury, renal function, glomerular function, and tubular function. In addition to the reduction of renal dysfunction (serum creatinine), administration of NaHS (15 min before ischemia and $5 \mathrm{~min}$ before reperfusion) also attenuated reperfusion injury (serum AST), renal injury (histology), glomerular dysfunction $(\mathrm{CCL})$, and tubular dysfunction $\left(\mathrm{FE}_{\mathrm{Na}+}\right)$ caused by renal IRI. What, then, is (are) the molecular mechanism(s) by which $\mathrm{H}_{2} \mathrm{~S}$ protects the kidney against IRI? At least two pathophysiological mechanisms lead to tubular cell death in ischemic renal injury. ${ }^{29-31}$ Necrosis is characterized by loss of membrane integrity, cytoplasmic swelling, nuclear pyknosis, cellular fragmentation, and an inflammatory response. Apoptosis is characterized by cytoplasmic and nuclear shrinkage, DNA fragmentation, and breakdown of the cell into apoptotic bodies that are rapidly removed by phagocytosis. Mounting evidence now indicates that apoptosis is the major mechanism of early tubular cell death in contemporary clinical acute kidney injury. Caspase-3 is one 

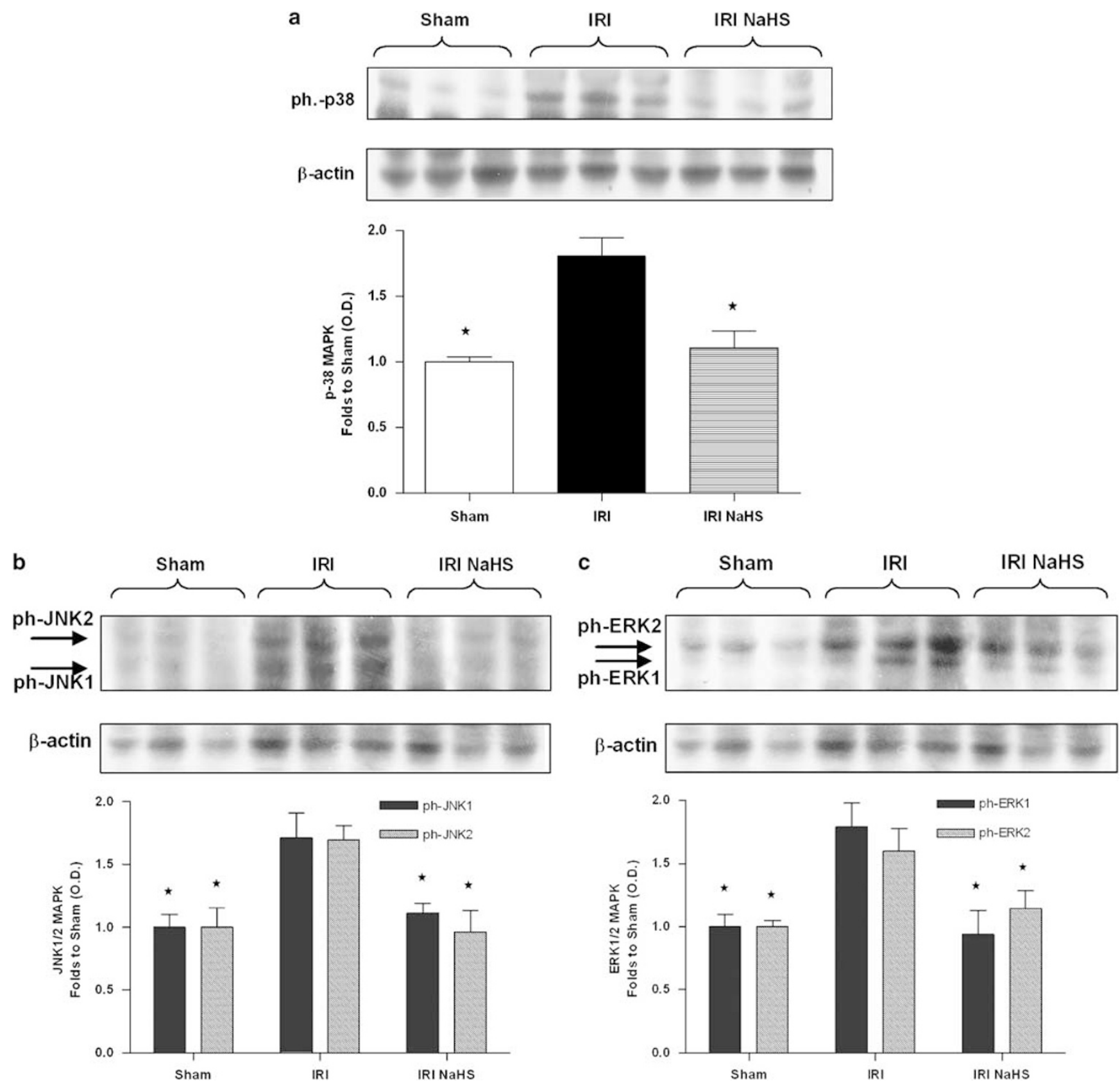

Figure 5 Exogenous $\mathrm{H}_{2} \mathrm{~S}$ prevents the increase in phosphorylation of mitogen-activated protein kinase $\mathrm{p}-38$, JNK1/2, and ERK1/2 caused by IRI in rats. Western blot analyses of phosphorylated (a) p-38, (b) JNK1/2, and (c) ERK1/2 in kidney homogenates from sham-operated rats (Sham), rats subjected to renal IRI (IRI), or rats subjected to renal IRI with NaHS $(100 \mu \mathrm{mol} / \mathrm{kg}, 2 \mathrm{ml} / \mathrm{kg}$, onto kidneys) administered $15 \mathrm{~min}$ before $45 \mathrm{~min}$ ischemia and $5 \mathrm{~min}$ before 30 min reperfusion (IRI NaHS). Densitometric analysis of the related bands is expressed as relative optical density (OD), corrected for the corresponding $\beta$-actin contents and normalized using the related sham-operated bands. Densitometric data are expressed as means \pm s.e.m. and each immunoblot is representative of three separate experiments. ${ }^{*} P<0.05$ vs IRI.

of the key executioners of apoptosis. Pancaspase (including caspase-3) inhibition prevents apoptosis in cold ischemic mouse kidney ${ }^{32}$ and the combination of caspase- 3 and complement 3 gene silencing prevents renal IRI. ${ }^{33}$ In addition, inhibition of caspase- 3 and -7 effectively reduces graft IRI and improves survival in liver transplantation. ${ }^{34}$ Thus, we have measured the activation of caspase- 3 in sections of the kidney as an indicator of the development of apoptosis. We provide clear evidence that $\mathrm{H}_{2} \mathrm{~S}$ attenuated the increase in renal caspase- 3 activation caused by IRI indicating that $\mathrm{H}_{2} \mathrm{~S}$ attenuates the formation of apoptosis caused by IRI. In addition, we have shown that Bid (a proapoptotic marker), which on activation translocates to mitochondria and induces damage to organelles, is activated during renal IRI 
a
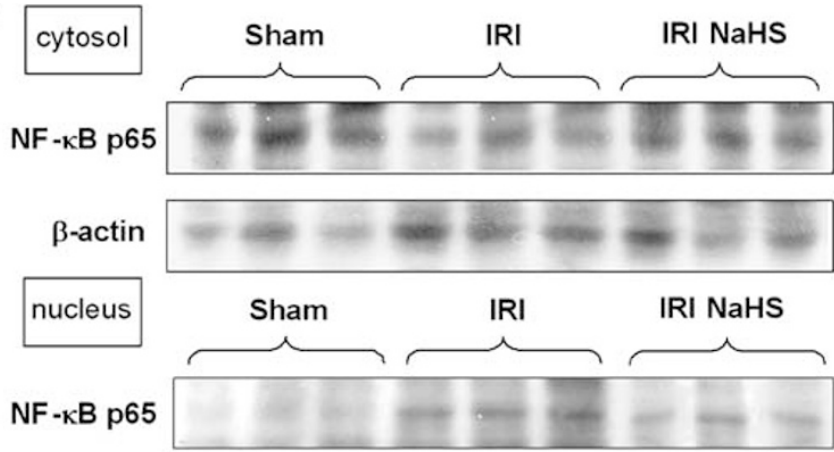

b

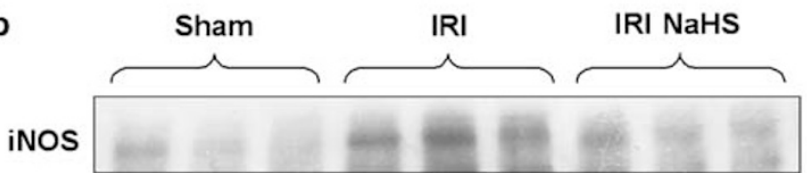

$\beta$-actin
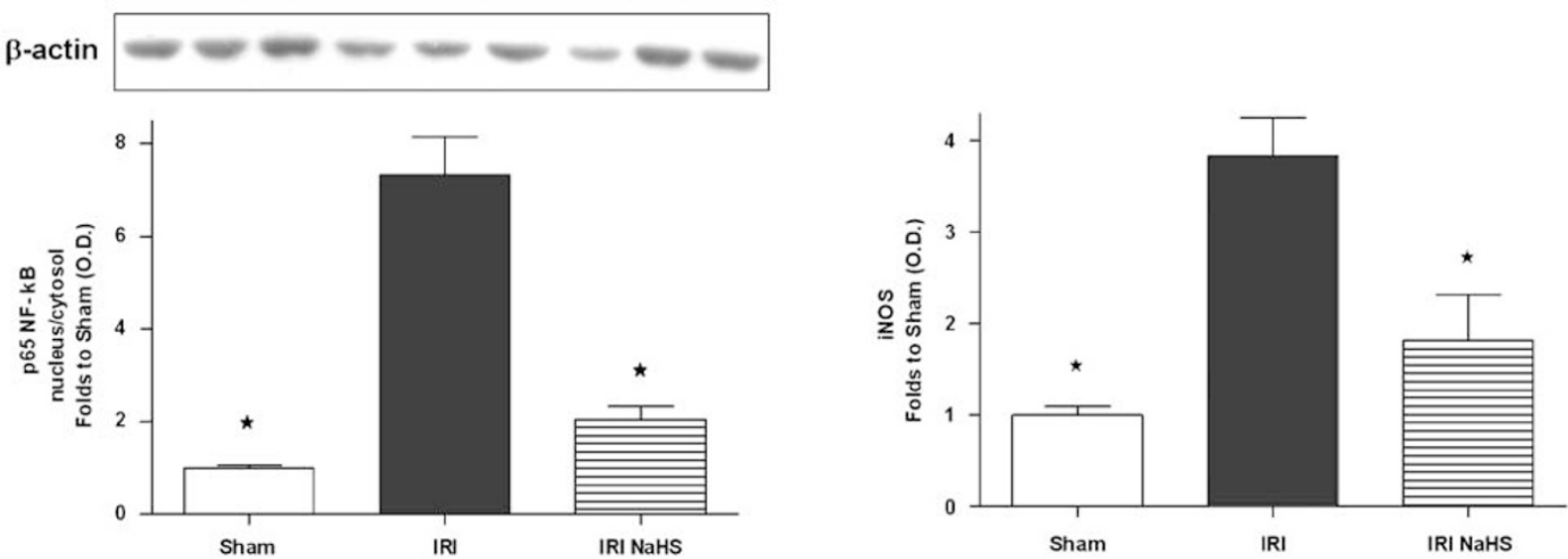

C

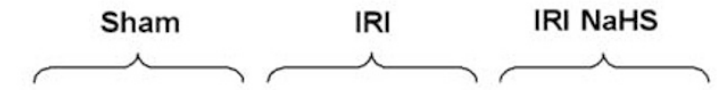

d
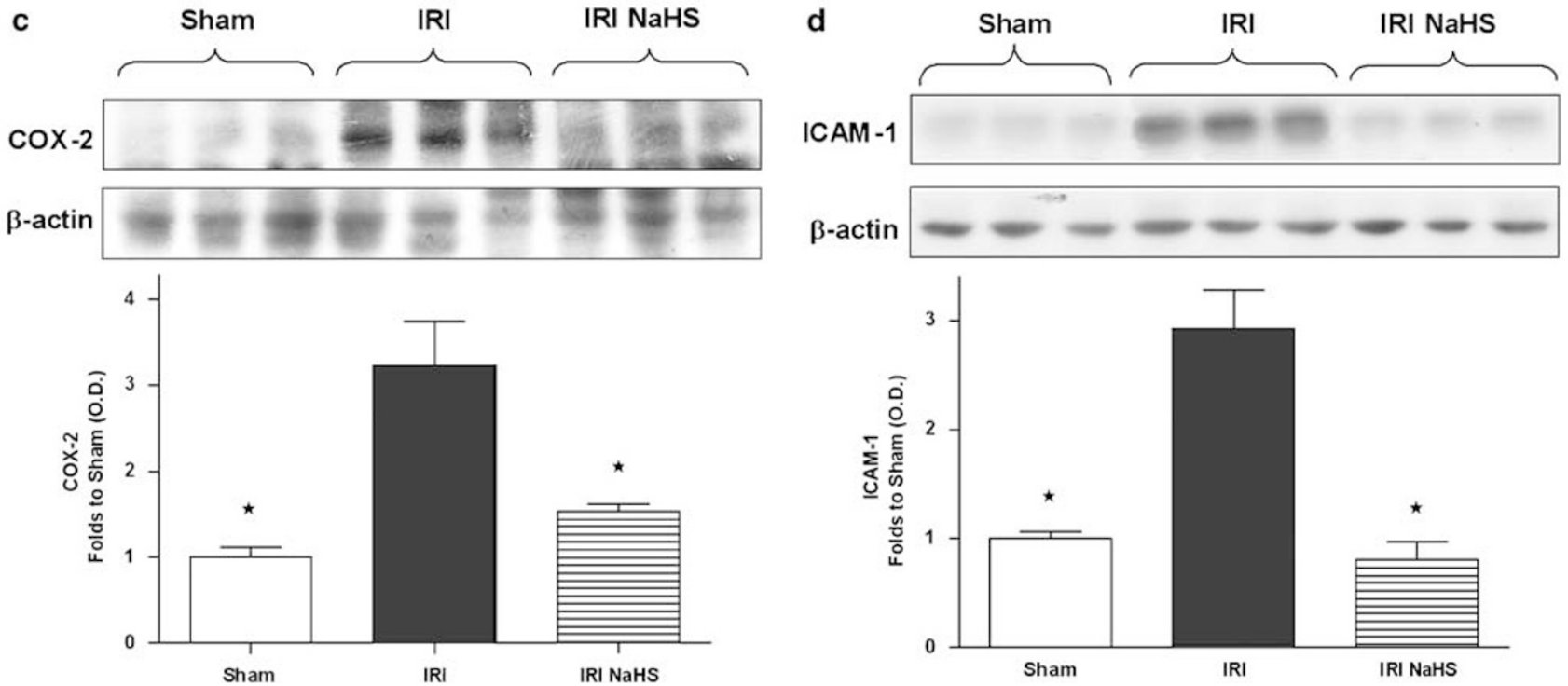

Figure 6 Exogenous $\mathrm{H}_{2} \mathrm{~S}$ prevents the nuclear translocation of the NF- $\kappa \mathrm{B}$ p65 subunit and the expression of iNOS, COX-2, and ICAM-1 caused by IRI in rats. (a) Western blot analyses of NF- $\kappa \mathrm{B}$ p65 subunit and $\beta$-actin expression in cytosolic and nuclear fractions in kidney homogenates from sham-operated rats (Sham), rats subjected to renal IRI (IRI), or rats subjected to renal IRI with NaHS (100 $\mu \mathrm{mol} / \mathrm{kg}, 2 \mathrm{ml} / \mathrm{kg}$, onto kidneys) administered $15 \mathrm{~min}$ before $45 \mathrm{~min}$ ischemia and $5 \mathrm{~min}$ before $30 \mathrm{~min}$ reperfusion (IRI NaHS). The results are expressed as nucleus/cytosol ratio of p65 NF- $\kappa \mathrm{B}$ from densitometric analysis of the related bands. Western blot analyses of (b) iNOS, (c) COX-2, and (d) ICAM-1 expression in kidney homogenates from sham-operated rats (Sham), rats subjected to renal IRI (IRI), or rats subjected to renal IRI with NaHS (100 $\mu \mathrm{mol} / \mathrm{kg}, 2 \mathrm{ml} / \mathrm{kg}$, onto kidneys) administered $15 \mathrm{~min}$ before $45 \mathrm{~min}$ ischemia and $5 \mathrm{~min}$ before $6 \mathrm{~h}$ reperfusion (IRI NaHS). Densitometric analysis of the related bands is expressed as relative optical density (OD), corrected for the corresponding $\beta$-actin contents and normalized using the related sham-operated bands. Densitometric data are expressed as means \pm s.e.m. and each immunoblot is representative of three separate experiments. ${ }^{*} P<0.05$ vs IRI. 
and is attenuated with NaHS administration. Activation of Bid depends on its proteolytic processing into truncated forms of tBid. Bid activation has previously been reported both in vivo following renal IRI in the rat and in vitro in primary cultures of rat proximal tubular cells following ATP depletion, ${ }^{35}$ which would be in agreement with our data. In addition, Bid cleavage has been shown to be abolished in cells overexpressing Bcl-2 (an antiapoptotic marker). ${ }^{35}$ Failure of $\mathrm{Bcl}-2$ upregulation in proximal tubular epithelial cells of donor kidney biopsy specimens is associated with apoptosis and delayed graft function, ${ }^{36}$ and augmentation of Bcl-2 protein inhibits proximal and distal tubular apoptosis and improved renal function. ${ }^{37}$ We have shown here that NaHS administration, which has exhibited protective effects in this study, prevented the decrease in Bcl-2 protein levels evoked by IRI.

It has been proposed that activation of MAPK p-38 and JNK contributes to cell death, whereas activation of ERK1/2 contributes to protection against cell injury in many organs. ${ }^{38,39}$ However, it has also been suggested that ERK1/2 activation may drive cell apoptosis in a number of cell types including renal epithelial cells. ${ }^{40}$ The importance of MAPKs in enhancing the tissue damage associated with IRI has been clearly demonstrated, ${ }^{41}$ and the activation of p-38, JNK and ERK1/2 is maximal within the first 30 min of reperfusion. ${ }^{42}$ Here, we confirm that ischemia for $45 \mathrm{~min}$ and reperfusion for 30 min results in activation (ie phosphorylation) of $\mathrm{p} 38$, JNK1/2 and ERK1/2 and, most notably, we show that these effects are attenuated by NaHS.

Several points of evidence suggest that MAPKs participate in the regulation of NF- $\kappa \mathrm{B}$ transcriptional activity. ${ }^{43}$ MAPK activation contributes to the development of the inflammatory process by modulating NF- $\kappa \mathrm{B}$ activity and transcriptional processes of proinflammatory genes. ${ }^{44}$ Activation of NF- $\kappa \mathrm{B}$ leads to the expression of numerous genes and mediators implicated in the development of renal IRI. Inhibitors of NF- $\kappa \mathrm{B}$ reduce the expression of iNOS, COX-2 and ICAM-1, and exert beneficial effects in various models of IRI. ${ }^{45-48}$ As we have demonstrated in this study that NaHS attenuates MAPK activation, we have conducted further experiments and found that NaHS attenuates the activation $\mathrm{NF}-\kappa \mathrm{B}$ (measured as nuclear translocation of p65) and consequently the expression of the NF- $\kappa \mathrm{B}$-dependent proteins iNOS, COX-2, and ICAM-1 caused by IRI in the kidney. Although $\mathrm{H}_{2} \mathrm{~S}$ has been reported to exhibit proinflammatory activities in endotoxic shock, ${ }^{17} \mathrm{H}_{2} \mathrm{~S}$ also has potent antiinflammatory effects. For instance, the $\mathrm{H}_{2} \mathrm{~S}$ donor ( $S$-diclofenac) downregulates the expression of iNOS, COX-2, CSE, and NF- $\kappa \mathrm{B}$ in a rat model of endotoxic shock. ${ }^{18}$ Thus, we propose that MAPK inhibition by NaHS is, at least in part, responsible for the antiapoptotic and anti-inflammatory effects of $\mathrm{H}_{2} \mathrm{~S}$ in our models of renal IRI. In addition, it is possible that NaHS interferes with the MAPK signaling cascade and, hence, affects NF- $\kappa$ B activation. However, the relationship between these signaling pathways in renal IRI still needs to be investigated further.
In conclusion, this article reports for the first time that (1) endogenous $\mathrm{H}_{2} \mathrm{~S}$ synthesis by CSE is essential to prevent further renal dysfunction and aids recovery of renal function and integrity following IRI, (2) exogenous $\mathrm{H}_{2} \mathrm{~S}$ protects the rat kidney against renal IRI, and (3) the observed beneficial effects of $\mathrm{H}_{2} \mathrm{~S}$ are due to both antiapoptotic and antiinflammatory effects of $\mathrm{H}_{2} \mathrm{~S}$, secondary to modulation of the signaling pathways leading to activation of MAPK and NF- $\kappa \mathrm{B}$. Thus, this study demonstrates for the first time a vital role of the formation of endogenous $\mathrm{H}_{2} \mathrm{~S}$ by CSE in IRI of the kidney, and implies that agents that release $\mathrm{H}_{2} \mathrm{~S}$ may be of benefit in conditions associated with renal IRI, such as renal transplantation.

\section{ACKNOWLEDGEMENTS}

PT is supported by a PhD studentship of Faculty of Medicine Siriraj Hospital, Mahidol University, Thailand. NSAP and this work are in part supported by the William Harvey Research Foundation, UK. Our special thanks to Professor Ken Suzuki, Dr Kenta Yashiro, and Dr Yasunori Shintani (Centre for Translational Medicine and Therapeutics, The William Harvey Research Institute, UK) for kind assistance with caspase-3 immunofluorescence.

\section{DISCLOSURE/DUALITY OF INTEREST}

There is no duality of interest to declare.

1. Wang R. Two's company, three's a crowd: can H2S be the third endogenous gaseous transmitter? FASEB J 2002;16:1792-1798.

2. Tvedt B, Skyberg $\mathrm{K}$, Aaserud $\mathrm{O}$, et al. Brain damage caused by hydrogen sulfide: a follow-up study of six patients. Am J Ind Med 1991;20:91-101.

3. Truong DH, Mihajlovic A, Gunness $\mathrm{P}$, et al. Prevention of hydrogen sulfide (H2S)-induced mouse lethality and cytotoxicity by hydroxocobalamin (vitamin B(12a)). Toxicology 2007;242:16-22.

4. Qu K, Lee SW, Bian JS, et al. Hydrogen sulfide: neurochemistry and neurobiology. Neurochem Int 2008;52:155-165.

5. $\mathrm{Qu} \mathrm{K}$, Chen $\mathrm{CP}$, Halliwell $\mathrm{B}$, et al. Hydrogen sulfide is a mediator of cerebral ischemic damage. Stroke 2006;37:889-893.

6. Sivarajah A, McDonald MC, Thiemermann C. The production of hydrogen sulfide limits myocardial ischemia and reperfusion injury and contributes to the cardioprotective effects of preconditioning with endotoxin, but not ischemia in the rat. Shock 2006;26:154-161.

7. Zhu YZ, Wang ZJ, Ho P, et al. Hydrogen sulfide and its possible roles in myocardial ischemia in experimental rats. J Appl Physiol 2007;102: 261-268.

8. Yong QC, Pan TT, Hu LF, et al. Negative regulation of beta-adrenergic function by hydrogen sulphide in the rat hearts. J Mol Cell Cardiol 2008:44:701-710.

9. Chunyu Z, Junbao D, Dingfang $B$, et al. The regulatory effect of hydrogen sulfide on hypoxic pulmonary hypertension in rats. Biochem Biophys Res Commun 2003;302:810-816.

10. Qingyou Z, Junbao D, Weijin Z, et al. Impact of hydrogen sulfide on carbon monoxide/heme oxygenase pathway in the pathogenesis of hypoxic pulmonary hypertension. Biochem Biophys Res Commun 2004;317:30-37.

11. Yan H, Du J, Tang $C$. The possible role of hydrogen sulfide on the pathogenesis of spontaneous hypertension in rats. Biochem Biophys Res Commun 2004;313:22-27.

12. Hui $Y, D u J$, Tang $C$, et al. Changes in arterial hydrogen sulfide $(H(2) S)$ content during septic shock and endotoxin shock in rats. J Infect 2003:47:155-160.

13. Mok YY, Atan MS, Yoke PC, et al. Role of hydrogen sulphide in haemorrhagic shock in the rat: protective effect of inhibitors of hydrogen sulphide biosynthesis. Br J Pharmacol 2004;143:881-889.

14. Collin M, Anuar FB, Murch $\mathrm{O}$, et al. Inhibition of endogenous hydrogen sulfide formation reduces the organ injury caused by endotoxemia. $\mathrm{Br}$ J Pharmacol 2005;146:498-505. 
15. Yang G, Wu L, Wang R. Pro-apoptotic effect of endogenous $\mathrm{H} 2 \mathrm{~S}$ on human aorta smooth muscle cells. FASEB J 2006;20:553-555.

16. Rinaldi $L$, Gobbi G, Pambianco $M$, et al. Hydrogen sulfide prevents apoptosis of human PMN via inhibition of p38 and caspase 3. Lab Invest 2006;86:391-397.

17. Li L, Bhatia M, Zhu YZ, et al. Hydrogen sulfide is a novel mediator of lipopolysaccharide-induced inflammation in the mouse. FASEB J 2005;19:1196-1198.

18. Li L, Rossoni G, Sparatore A, et al. Anti-inflammatory and gastrointestinal effects of a novel diclofenac derivative. Free Radic Biol Med 2007;42:706-719.

19. Hu LF, Wong PT, Moore PK, et al. Hydrogen sulfide attenuates lipopolysaccharide-induced inflammation by inhibition of p38 mitogen-activated protein kinase in microglia. J Neurochem 2007;100:1121-1128.

20. Beauchamp Jr RO, Bus JS, Popp JA, et al. A critical review of the literature on hydrogen sulfide toxicity. Crit Rev Toxicol 1984;13:25-97.

21. Blackstone $E$, Morrison M, Roth MB. H2S induces a suspended animation-like state in mice. Science 2005;308:518.

22. Watanabe M, Osada J, Aratani Y, et al. Mice deficient in cystathionine beta-synthase: animal models for mild and severe homocyst(e)inemia. Proc Natl Acad Sci USA 1995;92:1585-1589.

23. Thiemermann C, Patel NS, Kvale EO, et al. High density lipoprotein (HDL) reduces renal ischemia/reperfusion injury. J Am Soc Nephrol 2003;14:1833-1843.

24. Fiorucci S, Antonelli E, Distrutti E, et al. Inhibition of hydrogen sulfide generation contributes to gastric injury caused by anti-inflammatory nonsteroidal drugs. Gastroenterology 2005;129:1210-1224.

25. Chatterjee PK, Thiemermann C. An in vivo model of ischemia/ reperfusion and inflammation of the kidneys of the rat. Methods Mol Biol 2003;225:223-237.

26. Patel NS, Cortes U, Di PR, et al. Mice lacking the 110-kD isoform of poly(ADP-ribose) glycohydrolase are protected against renal ischemia/ reperfusion injury. J Am Soc Nephrol 2005;16:712-719.

27. Yashiro K, Shiratori H, Hamada H. Haemodynamics determined by a genetic programme govern asymmetric development of the aortic arch. Nature 2007;450:285-288.

28. Collino $M$, Aragno $M$, Mastrocola $R$, et al. Oxidative stress and inflammatory response evoked by transient cerebral ischemia/ reperfusion: effects of the PPAR-alpha agonist WY14643. Free Radic Biol Med 2006;41:579-589.

29. Saikumar $P$, Venkatachalam MA. Role of apoptosis in hypoxic/ischemic damage in the kidney. Semin Nephrol 2003;23:511-521.

30. Kaushal GP, Basnakian AG, Shah SV. Apoptotic pathways in ischemic acute renal failure. Kidney Int 2004;66:500-506.

31. Dagher PC. Apoptosis in ischemic renal injury: roles of GTP depletion and p53. Kidney Int 2004;66:506-509.

32. Jani $A$, Ljubanovic $D$, Faubel $S$, et al. Caspase inhibition prevents the increase in caspase- $3,-2,-8$ and -9 activity and apoptosis in the cold ischemic mouse kidney. Am J Transplant 2004;4: 1246-1254.
33. Zheng $X$, Zhang $X$, Sun $H$, et al. Protection of renal ischemia injury using combination gene silencing of complement 3 and caspase 3 genes. Transplantation 2006;82:1781-1786.

34. Mueller TH, Kienle K, Beham A, et al. Caspase 3 inhibition improves survival and reduces early graft injury after ischemia and reperfusion in rat liver transplantation. Transplantation 2004;78:1267-1273.

35. Wei $\mathrm{Q}$, Alam $\mathrm{MM}$, Wang $\mathrm{MH}$, et al. Bid activation in kidney cells following ATP depletion in vitro and ischemia in vivo. Am J Physiol Renal Physiol 2004;286:F803-F809.

36. Schwarz $C$, Hauser $P$, Steininger $R$, et al. Failure of $B C L-2$ up-regulation in proximal tubular epithelial cells of donor kidney biopsy specimens is associated with apoptosis and delayed graft function. Lab Invest 2002;82:941-948.

37. Chiang-Ting C, Tzu-Ching C, Ching-Yi T, et al. Adenovirus-mediated bcl2 gene transfer inhibits renal ischemia/reperfusion induced tubular oxidative stress and apoptosis. Am J Transplant 2005;5:1194-1203.

38. Xia Z, Dickens M, Raingeaud J, et al. Opposing effects of ERK and JNKp38 MAP kinases on apoptosis. Science 1995;270:1326-1331.

39. Park KM, Chen A, Bonventre JV. Prevention of kidney ischemia/ reperfusion-induced functional injury and JNK, p38, and MAPK kinase activation by remote ischemic pretreatment. J Biol Chem 2001;276:11870-11876.

40. Zhuang S, Yan Y, Daubert RA, et al. ERK promotes hydrogen peroxideinduced apoptosis through caspase-3 activation and inhibition of Akt in renal epithelial cells. Am J Physiol Renal Physiol 2007;292: F440-F447.

41. Irving EA, Bamford M. Role of mitogen- and stress-activated kinases in ischemic injury. J Cereb Blood Flow Metab 2002;22:631-647.

42. Yang $\mathrm{CW}$, Ahn $\mathrm{HJ}$, Jung JY, et al. Preconditioning with cyclosporine $\mathrm{A}$ or FK506 differentially regulates mitogen-activated protein kinase expression in rat kidneys with ischemia/reperfusion injury. Transplantation 2003;75:20-24.

43. Schulze-Osthoff K, Ferrari D, Riehemann K, et al. Regulation of NFkappa $B$ activation by MAP kinase cascades. Immunobiology 1997;198:35-49.

44. Kaminska B. MAPK signalling pathways as molecular targets for antiinflammatory therapy-from molecular mechanisms to therapeutic benefits. Biochim Biophys Acta 2005;1754:253-262.

45. McDonald MC, Mota-Filipe $H$, Paul A, et al. Calpain inhibitor I reduces the activation of nuclear factor-kappaB and organ injury/dysfunction in hemorrhagic shock. FASEB J 2001;15:171-186.

46. Chatterjee PK, Brown PA, Cuzzocrea S, et al. Calpain inhibitor-1 reduces renal ischemia/reperfusion injury in the rat. Kidney Int 2001;59: 2073-2083.

47. Chatterjee PK, di Villa Bianca RD, Sivarajah A, et al. Pyrrolidine dithiocarbamate reduces renal dysfunction and injury caused by ischemia/reperfusion of the rat kidney. Eur J Pharmacol 2003;482: 271-280.

48. Chatterjee PK, Patel NS, Cuzzocrea S, et al. The cyclopentenone prostaglandin 15-deoxy-Delta(12,14)-prostaglandin J2 ameliorates ischemic acute renal failure. Cardiovasc Res 2004;61:630-643. 